\title{
Clinical features and recurrent attack in gout patients according to serum urate levels during an acute attack
}

Jung Sun Lee, Oh Chan Kwon, Ji Seon Oh, Yong-Gil Kim, Chang-Keun Lee, Bin Yoo, and Seokchan Hong

Division of Rheumatology, Department of Internal Medicine, Asan Medical Center, University of Ulsan College of Medicine, Seoul, Korea

Received: June 13, 2018

Revised : July 14, 2018

Accepted: July 18, 2018

Correspondence to

Seokchan Hong, M.D.

Division of Rheumatology,

Department of Internal

Medicine, Asan Medical Center,

University of Ulsan College of

Medicine, 88 Olympic-ro 43-gil,

Songpa-gu, Seoul 05505, Korea

Tel: +82-2-3010-1410

Fax: +82-2-3010-6969

E-mail: medivineluke@gmail.com
Background/Aims: A substantial portion of gout patients have normal serum urate levels during an acute attack but data on the clinical characteristics and risk of recurrence compared with hyperuricemic patients are limited.

Methods: In this retrospective cohort study, clinical features of normouricemic and hyperuricemic patients were compared. Multivariate analysis was performed to determine whether normouricemic patients were less likely to have a recurrent attack.

Results: Among a total of 221 gout patients, 88 (39.8\%) had normouricemia during an acute attack. Postsurgical gout $(22.7 \%$ vs. $6.0 \%, p<0.001)$, hemodialysis initiation (9.1\% vs. $2.3 \%, p=0.029)$ and inflammatory activity were higher in normouricemic patients than in hyperuricemic patients. The frequency of renal insufficiency was lower in normouricemic patients ( $25.0 \%$ vs. $53.4 \%, p<0.001)$. However, the recurrence rate of gout attack was not different between the two groups $(24.7 \%$ vs. $33.0 \%, p=0.220$ ). In multivariate analysis, female sex, history of urinary stone, presence of tophi, and use of thiazide were associated with increase of recurrent gout attack, but not with serum urate status during an acute attack (hazard ratio, 1.075; 95\% confidence interval, 0.972 to $1.190 ; p=0.159$ ).

Conclusions: Normouricemia during an acute gout attack was more frequently observed in postsurgical episodes, hemodialysis initiation and patients with preserved renal function. While higher inflammatory activity was observed in normouricemic patients, recurrent attack was not associated with serum urate levels during an acute attack. Thus, careful follow-up should be considered in gout patients regardless of serum urate levels during an acute attack.

Keywords: Uric acid; Hyperuricemia; Gout; Inflammation; Recurrence

\section{INTRODUCTION}

Gout is characterized by joint inflammation resulting from the deposition of monosodium urate monohydrate. In hyperuricemia, defined as a serum urate level of $>7 \mathrm{mg} / \mathrm{dL}$ for men and $>6 \mathrm{mg} / \mathrm{dL}$ for women, the urate level exceeds the limit of solubility in the serum, and it is the most important risk factor for the development of gout. Therefore, the serum urate status is an essential element for the classification of gout [1].

Previous reports have shown that a substantial portion of patients with gout had serum urate levels within the normal range during an acute gout attack [2-5]. In these studies, normouricemia was found in $12 \%$ to $63.3 \%$ 
of gout patients during an acute gout attack. In addition, it was reported that serum urate levels were significantly decreased during an acute gout attack and that this is associated with an inflammatory process [6]. However, limited research has focused on what conditions are more frequently observed in normouricemia during an acute gout attack [7]. Furthermore, it is unknown whether there is a difference in the risk of subsequent attacks in patients with normouricemia compared with those with hyperuricemia. Therefore, in this study, we aimed to know the clinical characteristics of gout patients with normouricemia during an acute gout attack and addressed whether patients with normouricemia have different outcomes, including recurrence rate of further acute gout attack, during the follow-up period.

\section{METHODS}

\section{Study population}

We reviewed the electronic medical records of patients who were diagnosed with gout at Asan Medical Center, University of Ulsan College of Medicine, Seoul, Korea between January 2000 and December 2015. The diagnosis of gout was made during an acute gout attack, and it was confirmed by the presence of needle-shaped monosodium urate crystals on polarizing microscopy of the joint fluid. Normouricemia was defined as serum urate level $<7 \mathrm{mg} / \mathrm{dL}$ in men and $<6 \mathrm{mg} / \mathrm{dL}$ in women. Patients were divided into normouricemia and hyperuricemia groups according to their serum urate levels during an acute gout attack. Patients taking urate-lowering agents (including allopurinol, benzbromarone, and febuxostat) were excluded from the analysis. From the medical records, the following data were collected: (1) demographic information, including age, sex, and body mass index (BMI, $\mathrm{kg} / \mathrm{m}^{2}$ ); (2) data on accompanying medical conditions, such as hypertension, diabetes mellitus, renal insufficiency (defined as an estimated glomerular filtration rate of $<60 \mathrm{~mL} / \mathrm{min} / 1.73 \mathrm{~m}^{2}$ using the Chronic Kidney Disease Epidemiology Collaboration equation), coronary artery disease (CAD), active tuberculosis, transplantation, and stroke; (3) history of urinary stones and the presence of tophi; (4) medication (furosemide, thiazide, cyclosporine, ethambutol, pyrazinamide, aspirin or angiotensin II receptor blockers especially losartan or irbesartan); (5) predisposing factors such as surgery, infection, trauma, and initiation of hemodialysis; (6) number of involved joints; and (7) laboratory data, including white blood cell (WBC) counts, neutrophil counts, C-reactive protein (CRP; not high-sensitivity CRP), and serum urate levels during an acute gout attack. The results of the joint fluid analysis, including WBC counts, were analyzed. Postoperative acute gout attack was defined as an attack that developed within 3 weeks postoperatively. When it was available, previous and post urate levels were collected before and after 1 to 8 weeks from the initial acute gout attack. The diagnosis of a recurrent gout attack was made based on the 2015 or 1977 gout classification criteria $[1,8]$.

This study was approved by the Institutional Review Board of the Asan Medical Center, Seoul, Korea (IRB No.: 2016-1343). The requirement for informed consent was waived due to the retrospective nature of the study.

\section{Statistical analysis}

We analyzed the clinical features in patients with normouricemia and compared them to those of patients with hyperuricemia during an acute gout attack. The chi-square test and Fisher's exact test were used for comparisons of categorical data. Continuous values were expressed as mean $\pm \mathrm{SD}$ and median (interquartile range [IQR]) using the Student's $t$ test and the Mann-Whitney $U$ test, respectively, for comparing two independent groups of patients. Paired $t$ test was used to compare the differences uric acid levels between previous and during acute attack.

Cox regression analysis with backward elimination procedure was performed to identify risk factors for gout recurrence. Variables that had a $p<0.1$ on univariate analysis were selected for multivariate analysis. In analyzing the influence of the serum urate status on the development of gout recurrence, inverse probability of treatment weighting (IPTW) was used to exclude the influence of confounders. SPSS version 20.0 software (IBM Co., Armonk, NY, USA) was used for all statistical analysis. 


\section{RESULTS}

\section{Comparison of the normouricemia and hyperurice- mia groups during an acute gout attack}

Among a total of 221 patients with gout, 88 (39.8\%) had normouricemia during their acute gout attack (normouricemia group), whereas the remaining 133 (60.2\%) exhibited hyperuricemia during the acute gout attack (hyperuricemia group). Table 1 shows the baseline clinical characteristics and laboratory data of the patients with normouricemia and hyperuricemia. The mean age, mean BMI, and sex distribution were similar between the two groups. There was no significant difference in the presence of comorbidities, including hypertension, diabetes mellitus, and $\mathrm{CAD}$, between the two groups. However, patients with normouricemia were less likely to have renal insufficiency (22/88, 25.0\% vs. 71/133, 53.4\%; $p<0.001)$. Patients in the normouricemia group were less likely to have a history of urinary stones $(\mathrm{o} / 88,0.0 \%$ vs. $7 / 133,5.3 \% ; p=0.044)$ and use of furosemide $(5 / 88,5.7 \%$ vs. $25 / 133,18.8 \% ; p=0.005)$ than those in the hyperuricemia group. When we analyzed the factors associated with development of acute gout attack, the frequency of patients with postoperative gout $(20 / 88,22.7 \%$ vs. $8 / 133$, $6.0 \% ; p<0.001$ ) and hemodialysis initiation (8/88, $9.1 \%$ vs. $3 / 133,2.3 \% ; p=0.029)$ were significantly more frequent in normouricemia than in hyperuricemia. And, significantly higher inflammatory activity was observed in the patients in the normouricemia group than in those in the hyperuricemia group; normouricemia group had higher levels of joint fluid WBC counts, blood WBC counts, blood CRP, and higher rate of fever (Table 1).

Among a total of 221 patients, previous urate levels were available for $41 / 88(46.5 \%)$ in the normouricemia group and 50/133 (37.6\%) in the hyperuricemia group. In the normouricemia group, serum urate levels during an acute gout attack were significantly lower compared with serum urate levels before an acute gout attack (5.1 $\pm 1.3 \mathrm{mg} / \mathrm{dL}$ vs. $8.2 \pm 2.7 \mathrm{mg} / \mathrm{dL}, p<0.001$ ). In the hyperuricemia group, however, serum urate levels before and during the attack were not significantly different (10.2 \pm $2.7 \mathrm{mg} / \mathrm{dL}$ vs. $9.8 \pm 2.3 \mathrm{mg} / \mathrm{dL}, p=0.157$ ) (Fig. 1).

During the follow-up period, data were available for approximately $80 \%$ of both the groups (73/88 [83.0\%] in the normouricemia group and $115 / 133$ [86.5\%] in the hyperuricemia group) (Table 2). Among 188 patients, 56
(29.8\%) experienced at least one recurrent episode of acute gout attack. The median length of follow-up duration was 57 weeks (IQR, 10.5 to 207.5) and 59 weeks (IQR, 18 to 151$)$ in the normouricemia and hyperuricemia groups, respectively. There was no significant difference in the rate of initiation of urate-lowering therapy $(36 / 73$, $49.3 \%$ vs. $67 / 115,58.3 \%$; $p=0.230$ ). In addition, recurrence occurred in 18 patients $(24.7 \%)$ in the normouricemia group and 38 patients (33\%) in the hyperuricemia group, and this result was not significantly different between the two groups $(p=0.220)$.

\section{Clinical factors associated with gout recurrence}

Cox regression analysis was performed to evaluate which clinical factors were related to the recurrence of subsequent gout attack (Tables 3 and 4). On univariate analysis, female sex, history of urinary stones, presence of tophi, and use of furosemide or thiazide were significantly associated with the development of recurrent gout attack. In addition, serum urate level during the initial acute gout attack was marginally associated with the risk of recurrent subsequent attack (hazard ratio [HR], 1.102; 95\% confidence interval [CI], 1.003 to 1.211; $p=0.044$ ) (Table 3). Furthermore, in multivariate analysis, the following factors were associated with increased risk of recurrent gout attack: female sex (HR, 3.173; 95\% CI, 1.498 to 6.722; $\mathrm{p}=0.003)$, history of urinary stones (HR, 4.406; 95\% CI, 1.336 to 14.527; $p=0.015)$, presence of tophi (HR, 6.158; 95\% CI, 2.812 to $13.484 ; p<0.001)$, and use of thiazide (HR, 7.478; 95\% CI, 2.240 to 24.966 ; $p=0.001$ ). In contrast, the serum urate level during the initial attack was not associated with the increase of recurrent subsequent gout attack (HR, 1.075; 95\% CI, 0.972 to $1.190 ; p=0.159$ ) (Table 4). In addition, when we categorized the patients into normouricemia and hyperuricemia groups, no significant association for the risk of recurrent gout attack was observed (HR, 0.969; 95\% CI, 0.534 to 1.757; $p=0.916$ ). Finally, adjustment with the propensity score matching using IPTW confirmed that the risk of recurrent gout attack was not different for patients who had normouricemia and those who had hyperuricemia during the attack (IPTW adjusted HR, 0.815; 95\% CI, 0.363 to 1.828; $p$ $=0.62$ ). 
Lee JS, et al. Normouricemia in gout

Table 1. Comparison of baseline variables between patients with normouricemia and hyperuricemia during an acute gout attack

\begin{tabular}{|c|c|c|c|}
\hline Variable & Normouricemia $(\mathrm{n}=88)$ & Hyperuricemia $(\mathrm{n}=133)$ & $p$ value \\
\hline Age, yr & $61.6 \pm 11.6$ & $60.3 \pm 14.9$ & 0.450 \\
\hline Body mass index, $\mathrm{kg} / \mathrm{m}^{2}$ & $21.1 \pm 8.1$ & $22.7 \pm 16.6$ & 0.403 \\
\hline Male sex & $84(95 \cdot 5)$ & $120(90.2)$ & 0.153 \\
\hline \multicolumn{4}{|l|}{ Comorbidity } \\
\hline Hypertension & $44(50.0)$ & $52(39.1)$ & 0.109 \\
\hline Diabetes mellitus & $18(20.5)$ & $42(31.6)$ & 0.069 \\
\hline Renal insufficiency & $22(25 \cdot 0)$ & $71(53 \cdot 4)$ & $<0.001$ \\
\hline Coronary artery disease & $8(9.1)$ & $16(12.0)$ & 0.492 \\
\hline Tuberculosis & $2(2.3)$ & $4(3.0)$ & 1.000 \\
\hline Transplantation & $3(3.4)$ & $3(2.3)$ & 0.684 \\
\hline Stroke & $9(10.2)$ & $11(8.3)$ & 0.620 \\
\hline History of urinary stones & 0 & $7(5 \cdot 3)$ & 0.044 \\
\hline Presence of tophi & $6(6.9)$ & $7(5 \cdot 3)$ & 0.615 \\
\hline \multicolumn{4}{|l|}{ Drugs } \\
\hline Furosemide & $5(5 \cdot 7)$ & $25(18.8)$ & 0.005 \\
\hline Thiazide & $5(5 \cdot 7)$ & $5(3.8)$ & 0.524 \\
\hline Cyclosporine & $1(1.1)$ & $2(1.5)$ & 1.000 \\
\hline Ethambutol & $1(1.1)$ & $1(0.8)$ & 1.000 \\
\hline Pyrazinamide & $\mathrm{O}$ & $3(2.3)$ & 0.277 \\
\hline Aspirin & $14(15 \cdot 9)$ & $12(9.0)$ & 0.120 \\
\hline ARB & $8(9.1)$ & $13(9.8)$ & 0.865 \\
\hline \multicolumn{4}{|l|}{ Predisposing factor } \\
\hline Surgery & $20(22.7)$ & $8(6.0)$ & $<0.001$ \\
\hline Infection & $13(14.8)$ & $15(11.4)$ & 0.457 \\
\hline Trauma & $3(3.4)$ & $5(3.8)$ & 1.000 \\
\hline Hemodialysis initiation & $8(9.1)$ & $3(2.3)$ & 0.029 \\
\hline Articular involvement & & & $0.05^{8}$ \\
\hline Monoarticular & $57(64.8)$ & $89(66.9)$ & \\
\hline Oligoarticular & $30(34.1)$ & $34(25.6)$ & \\
\hline Polyarticular & $1(1.1)$ & $10(7 \cdot 5)$ & \\
\hline \multicolumn{4}{|l|}{ Joint fluid analysis } \\
\hline $\mathrm{WBC}, / \mu \mathrm{L}$ & $32,000(16,062-65,125)$ & $22,450(9,325-37,675)$ & 0.009 \\
\hline Neutrophil, / $\mu \mathrm{L}$ & $28,160(14,272-58,977)$ & $19,670(6,980-33,907)$ & 0.014 \\
\hline \multicolumn{4}{|l|}{ Blood laboratory data } \\
\hline $\mathrm{WBC}, / \mu \mathrm{L}$ & $10,680 \pm 4,452(\mathrm{n}=83)$ & $9,211 \pm 3,070(\mathrm{n}=128)$ & 0.010 \\
\hline Neutrophil, / $\mu \mathrm{L}$ & $8,041 \pm 4,143$ & $6,602 \pm 2,838$ & 0.006 \\
\hline $\mathrm{CRP}, \mathrm{mg} / \mathrm{dL}$ & $10.9(6.1-16.5)(n=77)$ & $6.3(2.2-12.2)(\mathrm{n}=111)$ & $<0.001$ \\
\hline $\mathrm{GFR}, \mathrm{mL} / \mathrm{min} / 1.73 \mathrm{~m}^{2}$ & $83.4(61.9-95.8)$ & $5^{2}(26.8-84.8)$ & $<0.001$ \\
\hline Urate during attack, mg/dL & $5.4(4.2-6.2)$ & $8.7(7.9-10.3)$ & $<0.001$ \\
\hline Fever & $44(50.0)$ & $45(33.8)$ & 0.016 \\
\hline
\end{tabular}

Values are presented as mean $\pm \mathrm{SD}$, number (\%), or median (interquartile range).

ARB, angiotensin II receptor blocker; WBC, white blood cell; CRP, C-reactive protein; GFR, glomerular filtration rate. 


\section{DISCUSSION}
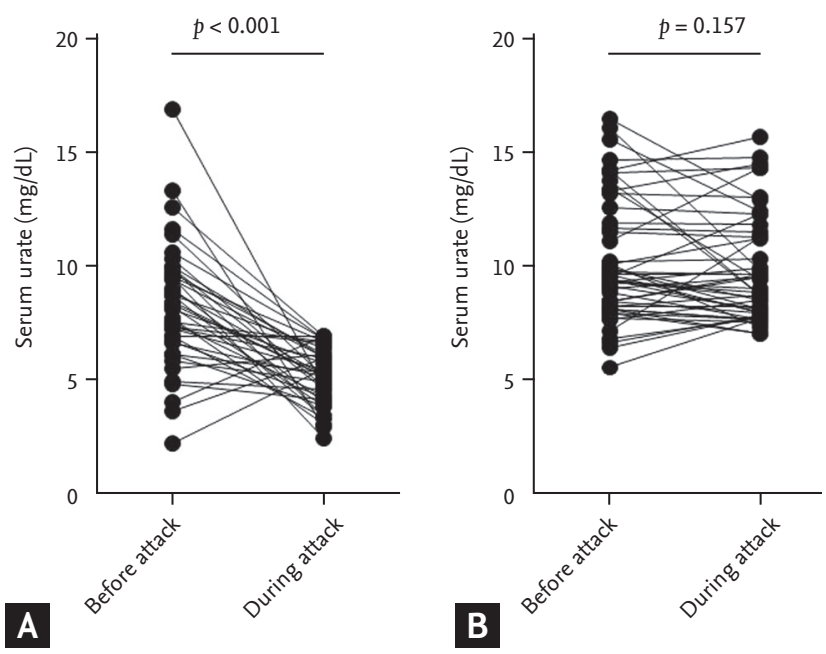

Figure 1. Change of serum urate levels before and during acute attack in (A) normouricemia and (B) hyperuricemia groups.
In the present study, the clinical features of patients with normouricemia were characterized by having more frequent postoperative episodes and hemodialysis initiation and less frequent renal insufficiency and urinary stones. In addition, more pronounced inflammation, with fever and elevated WBC counts and CRP levels, was observed in the normouricemia group than in the hyperuricemia group. However, serum urate levels during an acute gout attack were not associated with recurrent gout attack.

Surgery has been known to be a risk factor for acute gout attack, related to starvation, catabolism, or dehydration [9]. Interestingly, a previous research has shown that serum urate levels of patients with postoperative gout are significantly lower when compared with the preoperative serum urate levels [10]. Moreover, in our study, serum urate levels of patients with postoperative acute gout attack were significantly lower during the acute gout attack $(6.23 \pm 2.87 \mathrm{mg} / \mathrm{dL})$ than before the

Table 2. Outcomes of gout patients with normouricemia and hyperuricemia

\begin{tabular}{lccc}
\hline Variable & Normouricemia $(\mathrm{n}=73)$ & Hyperuricemia $(\mathrm{n}=115)$ & $p$ value \\
\hline Follow-up time, wk & $57(10.5-207.5)$ & $59(18-151)$ & 0.707 \\
Initiation of urate-lowering therapy & $36(49.3)$ & $67(58.3)$ & 0.230 \\
Time to recurrence, wk & $11.5(6.8-32.0)$ & $18(7.8-43.3)$ & 0.276 \\
Recurrence rate & $18(24.7)$ & $38(33.0)$ & 0.220 \\
\hline
\end{tabular}

Values are presented as median (interquartile range) or number (\%).

Table 3. Univariate analysis of variables associated with recurrence of gout

\begin{tabular}{lccc}
\hline Variable & HR & $95 \%$ CI & p value \\
\hline Female sex & 2.864 & $1.397-5.873$ & 0.004 \\
History of urinary stones & 3.573 & $1.106-11.543$ & 0.033 \\
Presence of tophi & 5.160 & $2.401-11.090$ & $<0.001$ \\
Use of furosemide & 2.067 & $1.040-4.107$ & 0.038 \\
Use of thiazide & 5.539 & $1.710-17.939$ & 0.004 \\
Use of ARB & 0.801 & $0.289-2.217$ & 0.669 \\
Alcohol intake & 1.405 & $0.825-2.393$ & 0.211 \\
Diabetes mellitus & 1.215 & $0.963-2.129$ & 0.497 \\
Renal insufficiency & 1.524 & $0.902-2.575$ & 0.115 \\
Serum urate level during attack & 1.102 & $1.003-1.211$ & 0.044 \\
\hline
\end{tabular}

HR, hazard ratio; CI, confidence interval; ARB, angiotensin II receptor blocker. 
Table 4. Multivariate analysis of variables associated with recurrence of gout

\begin{tabular}{llcr}
\hline Variable & HR & 95\% CI & $p$ value \\
\hline Female sex & 3.173 & $1.498-6.722$ & 0.003 \\
History of urinary stones & 4.406 & $1.336-14.527$ & 0.015 \\
Presence of tophi & 6.158 & $2.812-13.484$ & $<0.001$ \\
Use of thiazide & 7.478 & $2.240-24.966$ & 0.001 \\
Serum urate level during attack & 1.075 & $0.972-1.190$ & 0.159 \\
\hline
\end{tabular}

HR, hazard ratio; CI, confidence interval.

acute gout attack $(8.99 \pm 2.46 \mathrm{mg} / \mathrm{dL})(p<0.001)$. In addition, postoperative acute gout attacks occur more often after gastrointestinal surgery than after other types of surgeries [10]. Indeed, in the present study, patients with normouricemia more frequently underwent gastrointestinal surgery than those with hyperuricemia, although this finding was not statistically significant (11/20, $55 \%$ vs. $1 / 8,12.5 \% ; p=0.088)$. Several factors, such as administration of anesthetics, intestinal manipulation, and intravenous infusion of glucose or total parenteral nutrition, are associated with a marked increase in urinary excretion of serum urate during the postoperative period following abdominal surgery [11-14].

Serum urate reduction induce partial dissolution of tophi and released uric acid can activate a cascade of pro-inflammatory cytokines [15]. Previous studies reported the development of acute gout attack after initiation of dialysis $[16,17]$. In our present study, normouricemia during acute attack was more frequently observed in patients with hemodialysis initiation (Table 1); therefore, this can be explained by a rapid reduction of serum urate levels by hemodialysis.

Our findings showed that inflammatory activity, as assessed by the presence of fever and elevated CRP levels and WBC counts in the blood or joint fluid, was significantly higher in the normouricemia group than in the hyperuricemia group. A previous research found that the inflammatory process can play a role in the reduction of serum urate levels during an acute gout attack through urinary excretion of uric acid [6]. It has been suggested that proinflammatory cytokines can induce the release of cortisol, which has uricosuric effect [7,18]. In the present study, the patients in the normouricemia group had significantly lower serum urate levels during an acute gout attack compared with previous serum urate levels (Fig. 1). Thus, we can speculate that more inflammatory activity underlies the mechanisms responsible for the reduction of serum urate levels to be within the normal range.

A significantly lower rate of renal insufficiency was observed in the patients in the normouricemia group than in those in the hyperuricemia group (Table 1). There are several factors that influence the development of hyperuricemia in renal function decline. These include decreased delivery of a filtered urate load to the renal tubule and increased reabsorption and/or decreased tubular secretion of uric acid [19]. Recent studies have reported that urate transporters in the renal proximal tubule act on reabsorption and secretion of uric acid [20-22]. However, in patients with renal impairment, uric acid handling by renal urate transporters can be compromised and extrarenal urate transporters may play a greater role in determining serum urate levels $[23,24]$. Therefore, urate urinary excretion under certain conditions, such as those of surgery and inflammation, could occur more actively and efficiently in patients with normal kidney function than in those with renal insufficiency during an acute gout attack. This hypothesis might explain why normouricemia status is less likely to occur in patients with renal insufficiency.

The present study has shown that female sex is a risk factor for gout recurrence. Female hormone is associated with increased urinary excretion of uric acid; therefore, females are not generally affected by hyperuricemia before menopause [25]. However, serum urate concentrations sharply increase after menopause, which leads to increased incidence of gout in postmenopausal women [26]. Female patients with gout were found to be older, had more comorbidities including hypertension or renal insufficiency, and received diuretics more 
often $[27,28]$. In a previous study, hypertension, renal insufficiency, and use of diuretics were identified as risk factors of recurrent gout attacks [29]. However, the effect of sex differences as well as hormones regarding the recurrence of gout attacks remain unclear. Therefore, it would be interesting to discover which factors are responsible for the elevated risk of gout recurrence in female patients.

In the present study, the presence of tophi and history of urinary stone were associated with the recurrence of gout attack. A previous study showed that serum urate levels were correlated with the number of tophi [30]. In addition, serum urate levels act as a risk factor of urinary stone in a dose dependent manner [31]. Thus, high urate burden in the presence of tophi or history of urinary stone may contribute to the recurrence of acute gout attack.

Diuretics increase serum urate levels by volume depletion and decrease in renal tubular secretion of uric acid [32]. In a previous study, the use of loop diuretics or thiazide were associated with the incidence of gout attack [33]. In addition, recent use of diuretics, especially thiazide, is an important risk factor for recurrent gout attack [34]. Similarly, in our present study, the use of thiazide was associated with subsequent gout attacks.

Hyperuricemia has been reported to be closely linked to the development and recurrence of gout attack; thus, the reduction of serum urate levels is important for the reduction of future acute gout attacks [35]. However, it is not well known whether normouricemia during an acute gout attack is associated with a lower risk of subsequent gout attack. In the present study, our finding showed that serum urate level during an acute gout attack was not associated with a different risk of subsequent gout attack as assessed by multivariate analysis (Table 2) and IPTW analysis. Furthermore, the rate of initiation of a urate-lowering agent was not significantly different between the normouricemia and hyperuricemia groups. According to a previous study, $81 \%$ of gout patients with normouricemia at diagnosis subsequently developed hyperuricemia [3]. In the present study, serum urate levels obtained both before and after the acute gout attack were available for 32 patients in the normouricemia group. Among these, only six patients (18.8\%) showed persistent normouricemia, whereas the remaining $26(81.3 \%)$ showed hyperuricemia at some point before and/or after the acute gout attack. These findings suggest that normouricemia during an acute gout attack may be a transient phenomenon of urate levels and is not associated with a lower risk of subsequent gout attack.

The present study has some limitations. Due to the retrospective design of the study, we could not analyze dietary food and the exact amount of alcohol intake, which can affect recurrent gout attacks and exclude the possibility that acute gout attack was not detected if the patients did not visit the clinic and some data may be missing. In addition, our findings have been obtained in patients with crystals confirmed by polarizing microscopy of the aspirated fluid that allow us to diagnose exactly in patients with normouricemia during acute attack, but the study population tended to be having a large-joint involvement thus it cannot be generalised.

In conclusion, approximately $40 \%$ patients with gout had normouricemia during an acute attack and the clinical features of these patients were characterized by a higher rate of postsurgical episodes, hemodialysis initiation, and inflammatory activity and a lower rate of renal insufficiency. The recurrence of subsequent gout attack was significantly associated with female sex, history of urinary stones, presence of tophi, and use of thiazide, but not with serum urate levels during the initial acute gout attack. Thus, careful diagnosis and follow-up should be considered in patients with gout symptoms regardless of serum urate levels during an acute gout attack.

\section{KEY MESSAGE}

1. Normouricemia during an acute attack occurs more frequently after surgery and at hemodialysis initiation.

2. It is associated with higher inflammation levels.

3. Normouricemia during acute attack has a similar risk in the subsequent recurrence of gout.

\section{Conflict of interest}

No potential conflict of interest relevant to this article was reported. 


\section{Acknowledgments}

This work was supported by a grant from the Korea Health Technology R\&D Project through the Korea Health Industry Development Institute, funded by the Ministry of Health and Welfare, Republic of Korea (grant number: $\mathrm{HI}_{15} \mathrm{C}_{3117}$ ).

\section{REFERENCES}

1. Neogi T, Jansen TL, Dalbeth N, et al. 2015 Gout classification criteria: an American College of Rheumatology/ European League Against Rheumatism collaborative initiative. Arthritis Rheumatol 2015;67:2557-2568.

2. Badulescu M, Macovei L, Rezus E. Acute gout attack with normal serum uric acid levels. Rev Med Chir Soc Med Nat Iasi 2014;118:942-945.

3. Park YB, Park YS, Lee SC, Yoon SJ, Lee SK. Clinical analysis of gouty patients with normouricaemia at diagnosis. Ann Rheum Dis 2003;62:90-92.

4. Sc hlesinger N, Norquist JM, Watson DJ. Serum urate during acute gout. J Rheumatol 2009;36:1287-1289.

5. Schlesinger N, Schumacher HR Jr, Beutler AM. Serum uric acid in acute gout. Ann Rheum Dis 1998;57:443-444.

6. Urano W, Yamanaka H, Tsutani H, et al. The inflammatory process in the mechanism of decreased serum uric acid concentrations during acute gouty arthritis. J Rheumatol 2002;29:1950-1953.

7. McCarty DJ. Gout without hyperuricemia. JAMA 1994; 271:302-303.

8. Wallace SL, Robinson H, Masi AT, Decker JL, McCarty DJ, Yu TF. Preliminary criteria for the classification of the acute arthritis of primary gout. Arthritis Rheum 1977;20:895-900.

9. Craig MH, Poole GV, Hauser CJ. Postsurgical gout. Am Surg 1995;61:56-59.

10. Kang EH, Lee EY, Lee YJ, Song YW, Lee EB. Clinical features and risk factors of postsurgical gout. Ann Rheum Dis 2008;67:1271-1275.

11. Diamond HS, Lazarus R, Kaplan D, Halberstam D. Effect of urine flow rate on uric acid excretion in man. Arthritis Rheum 1972;15:338-346.

12. Masuda A, Asahi T, Sakamaki M, Nakamaru K, Hirota K, Ito $Y$. Uric acid excretion increases during propofol anesthesia. Anesth Analg 1997;85:144-148.

13. Moyer RA, John DS. Acute gout precipitated by total par- enteral nutrition. J Rheumatol 2003;30:849-850.

14. Snaith ML, Scott JT. Uric acid excretion and surgery. Ann Rheum Dis 1972;31:162-165.

15. Liu-Bryan R, Scott P, Sydlaske A, Rose DM, Terkeltaub R. Innate immunity conferred by Toll-like receptors 2 and 4 and myeloid differentiation factor 88 expression is pivotal to monosodium urate monohydrate crystal-induced inflammation. Arthritis Rheum 2005:52:2936-2946.

16. Iwata N, Maeda H, Yoshimura J, Sakai S. Urgent hemodialysis induced an acute gout attack in a patient with multiple tophi: report of a rare case. CEN Case Rep 2012;1:130131.

17. Ohno I, Ichida K, Okabe H, et al. Frequency of gouty arthritis in patients with end-stage renal disease in Japan. Intern Med 2005;44:706-709.

18. Dunn AJ. Cytokine activation of the HPA axis. Ann N Y Acad Sci 2000;917:608-617.

19. Gibson T, Highton J, Potter C, Simmonds HA. Renal impairment and gout. Ann Rheum Dis 1980;39:417-423.

20. Bobulescu IA, Moe OW. Renal transport of uric acid: evolving concepts and uncertainties. Adv Chronic Kidney Dis 2012;19:358-371.

21. Hyndman D, Liu S, Miner JN. Urate handling in the human body. Curr Rheumatol Rep 2016;18:34.

22. Pascual E, Perdiguero M. Gout, diuretics and the kidney. Ann Rheum Dis 2006;65:981-982.

23. Bhatnagar V, Richard EL, Wu W, et al. Analysis of ABCG2 and other urate transporters in uric acid homeostasis in chronic kidney disease: potential role of remote sensing and signaling. Clin Kidney J 2016;9:444-453.

24. Yano H, Tamura Y, Kobayashi K, Tanemoto M, Uchida S. Uric acid transporter ABCG2 is increased in the intestine of the 5/6 nephrectomy rat model of chronic kidney disease. Clin Exp Nephrol 2014;18:50-55.

25. Marinello E, Riario-Sforza G, Marcolongo R. Plasma follicle-stimulating hormone, luteinizing hormone, and sex hormones in patients with gout. Arthritis Rheum 1985;28:127-131.

26. Suresh E. Diagnosis and management of gout: a rational approach. Postgrad Med J 2005;81:572-579.

27. Dirken-Heukensfeldt KJ, Teunissen TA, van de Lisdonk $\mathrm{H}$, Lagro-Janssen AL. "Clinical features of women with gout arthritis." A systematic review. Clin Rheumatol 2010;29:575-582.

28. Harrold LR, Yood RA, Mikuls TR, et al. Sex differences in gout epidemiology: evaluation and treatment. Ann 
Rheum Dis 2006;65:1368-1372.

29. Rothenbacher D, Primatesta P, Ferreira A, Cea-Soriano L, Rodriguez LA. Frequency and risk factors of gout flares in a large population-based cohort of incident gout. Rheumatology (Oxford) 2011;50:973-981.

30. Khanna PP, Nuki G, Bardin T, et al. Tophi and frequent gout flares are associated with impairments to quality of life, productivity, and increased healthcare resource use: Results from a cross-sectional survey. Health Qual Life Outcomes 2012;10:117.

31. Kim S, Chang Y, Yun KE, et al. Development of nephrolithiasis in asymptomatic hyperuricemia: a cohort study. Am J Kidney Dis 2017;70:173-181.

32. Kahn AM. Effect of diuretics on the renal handling of urate. Semin Nephrol 1988;8:305-314.

33. Bruderer S, Bodmer M, Jick SS, Meier CR. Use of diuretics and risk of incident gout: a population-based case-control study. Arthritis Rheumatol 2014;66:185-196.

34. Hunter DJ, York M, Chaisson CE, Woods R, Niu J, Zhang Y. Recent diuretic use and the risk of recurrent gout attacks: the online case-crossover gout study. J Rheumatol 2006;33:1341-1345.

35. Shoji A, Yamanaka H, Kamatani N. A retrospective study of the relationship between serum urate level and recurrent attacks of gouty arthritis: evidence for reduction of recurrent gouty arthritis with antihyperuricemic therapy. Arthritis Rheum 2004;51:321-325. 